\title{
Theoretical band alignment in an intermediate band chalcopyrite based material
}

\author{
J.E. Castellanos Águila ${ }^{\mathrm{a}, \mathrm{d}}$, P. Palacios ${ }^{\mathrm{a}, \mathrm{b}, *}$, J.C. Conesa $^{\mathrm{e}}$, J. Arriaga ${ }^{\mathrm{d}}$, P. Wahnón ${ }^{\mathrm{a}, \mathrm{c}}$ \\ a Instituto de Energía Solar, Universidad Politécnica de Madrid, 28040 Madrid, Spain \\ b Dpt. Física Aplicada a las Ingenierías Aeronáutica y Naval, Universidad Politécnica de Madrid, ETSI Aeronáutica y del Espacio, 28040 Madrid, Spain \\ c Dpt. Tecnología Fotónica y Bioingeniería, Universidad Politécnica de Madrid, ETSI Telecomunicación, 28040 Madrid, Spain \\ d Instituto de Física, Benemérita Universidad Autónoma de Puebla, Av. San Claudio y 18 Sur, C.U. 72570 Puebla, Mexico \\ e Instituto de Catálisis y Petroleoquímica, CSIC, Marie Curie 2, Cantoblanco, 28049 Madrid, Spain
}

\section{A R T I C L E I N F O}

\section{Article history:}

Received 25 October 2016

Received in revised form

29 December 2016

Accepted 30 December 2016

Available online $\mathrm{xxx}$

\section{Keywords:}

Photovoltaics

Theoretical calculations

Heterostructures

\begin{abstract}
A B S T R A C T
Band alignment is key to enhance the performance of heterojunction for chalcopyrite thin film solar cells. In this paper we report ab initio calculations of the electronic structures of $\mathrm{CuGaS}_{2}: \mathrm{Cr}$ with various $\mathrm{Cr}$ compositions, CuAlSe ${ }_{2}$ and $\mathrm{ZnSe}$ and the band alignment between their interfaces. We use density functional theory and the more accurate self-consistent GW scheme to obtain improved bulk band-gaps and band offsets. Band alignments of the interfacial region for $\mathrm{CuGaS}_{2}: \mathrm{Cr} / \mathrm{CuAlSe}_{2}$ and $\mathrm{CuGaS}_{2}: \mathrm{Cr} / \mathrm{ZnSe}$ systems were aligned with respect of an average electrostatic potential. Our results are in good agreement with experimental values for the bulk band-gaps. These theoretical band alignments show a characteristic staggered band alignment for the design of heterojunction devices in photovoltaic applications.
\end{abstract}

(C) 2016 Elsevier B.V. All rights reserved.

\section{Introduction}

Current $\mathrm{Cu}(\mathrm{In}, \mathrm{Ga}) \mathrm{Se}_{2}$ photovoltaics devices, on a laboratory scale, reach conversion efficiencies about $22.3 \%$ [1]. This improvement on the efficiency is mainly due to the improvement to the CIGS absorber layer and the junction formation process. However, the efficiency is still lower than the single gap Shockley-Queisser limit [2]. One of the proposals which promises high conversion efficiencies is the intermediate band (IB) solar cells concept [3]. An IB could provide additional optical transitions due to electronic states inside the fundamental band-gap of the host semiconductor. It has been reported that the $\mathrm{CuGaS}_{2}$ which has a $2.43 \mathrm{eV}$ band-gap, is a suitable host material for the IB concept $[4,5]$. If we consider Chromium transition atom replacing Gallium atom in a $\mathrm{CuGaS}_{2}$ semiconductor, additional states within the band-gap are observed due to the $\mathrm{Cr}$, as described in Ref. [6-10]. However, up to date it is not known which will be the behaviour of the intermediate band material taking into account the rest of materials which are in contact in the complete solar cell. For this reason, a precise knowledge of the band structure of these heterojunctions becomes necessary.

\footnotetext{
* Corresponding author at: Dpt. Física Aplicada a las Ingenierías Aeronáutica y Naval, UPM, ETSI Aeronáutica y del Espacio, 28040, Madrid.

E-mail address: pablo.palacios@upm.es (P. Palacios).
}

In a previous report [11], we have studied the band alignment of the heterointerfaces between $\mathrm{CuGaS}_{2}$ and several semiconductors. We have found that the $\mathrm{CuAlSe}_{2} / \mathrm{CuGaS}_{2}$ and $\mathrm{CuGaS}_{2} / \mathrm{ZnSe}$ heterointerfaces show good characteristics for the design and development of thin film solar cells. The junctions formed by materials with different band-gaps and lattice constants have strong influence on the valence and conduction band offsets, and moreover the possibility that local states can occur at the interfaces of these heterostructures which might have an influence in the electronic and transport properties. A proper description of the structure and electronic properties of surfaces and heterointerfaces must be taken into account in order to design, to build and to analyze such heterostructures devices.

In this work, we present a systematic procedure for the calculation of the energy band alignment at an abrupt IB materialsemiconductor heterojunction $[12,13]$. The procedures requires only a knowledge of the energy band structures of the participating semiconductors, and does not invoke any properties - empirical or theoretical - of the free surface, but needs to associate the energy levels of the semiconductors which comprise the heterostructure with a common reference energy level. This level is obtained using an average electrostatic potential. The accuracy of the method is believed to reflect directly the quality of the band structures. Once accurate band structures and an adequate sophisticated model of the charge distribution near the interface are obtained, the method should be capable of serving as an accurate tool for the 
quantitative prediction of band lineups at heterojunctions for which no experimental data yet exist.

\section{Model and computational technique}

We perform first-principles calculations based on the GGA approximation [14] and many body perturbation theory (quasiparticle energy calculations) $[15,16]$ as implemented in the Vienna ab initio simulation package (VASP) [17]. The interactions between the ionic cores and the valence electrons were introduced using the projector-augmented wave method (PAW) [18,19]. The valence configurations used in the PAW pseudopotentials were: $3 \mathrm{~d}^{10} 4 \mathrm{~s}^{1}$ for $\mathrm{Cu} ; 4 \mathrm{~s}^{2} 4 \mathrm{p}^{1}$ for $\mathrm{Ga} ; 3 \mathrm{~s}^{2} 3 \mathrm{p}^{4}$ for $\mathrm{S}, 3 \mathrm{~d}^{5} 4 \mathrm{~s}^{1}$ for $\mathrm{Cr}, 4 \mathrm{~s}^{2} 4 \mathrm{p}^{4}$ for $\mathrm{S}, 3 \mathrm{~s}^{2} 3 \mathrm{p}^{1}$ for $\mathrm{Al}$ and $4 \mathrm{~s}^{2} 3 \mathrm{~d}^{10}$ for $\mathrm{Zn}$. Ga potential without semicore $d$ states reproduces quite well the experimental valence band levels in $\mathrm{CuGaS}_{2}$ semiconductor so they have not been included. The Perdew-Burke-Ernzerhof (PBE) [14] functionals are employed for the GGA exchange-correlation potential. The valence electronic wave-functions are expanded in a plane wave basis set up to a kinetic energy cut-off of $450 \mathrm{eV}$. The quasiparticle energy calculations are based on a restricted self-consistent scGW schemes, where, the wave-functions and energies of the PBE calculations were used as the starting point to compute the quasiparticle band structure, subsequently, we update the quasiparticle wave-function four times in both Green's function $G$ and screened potential $W[20]$. The total number of valence and conduction band states in the scGW procedure was set to 320 for all materials.

To calculate the band alignment from first principles calculations we use non-polar interfaces because their structures are relatively well established. The heterointerfaces between the IB material and $\mathrm{CuAlSe}_{2}$ or ZnSe phases are studied using a typical supercell approach. Basically a supercell consists of a unit cell of $n$ monolayers of one semiconductor followed by $m$ monolayers of the other. Eight atomic layers for each of the two materials are stacked in the [110] direction. Supercells composed of 16 layers, correspond to a $\sim 32 \AA$ A thickness. Here, $\Gamma$-centred $k$-point Monkhorst-Pack [21] mesh of $6 \times 6 \times 1$ was used, due to the relatively large supercell size. For the bulk calculations we use a $6 \times 6 \times 2 k$-point mesh. The plane wave cut-off and the Brillouin zone sampling both were verified to provide convergence in total lattice energy within $1 \mathrm{meV}$. The calculation for $\mathrm{CuGaS}_{2}$ : $\mathrm{Cr}$ was carried out using a monoclinic unit cell in which one of the Ga atoms was replaced by a $\mathrm{Cr}$ atom (at tetrahedral sites), which corresponds to the $25 \%$ dopant concentration.

Two types of calculations were performed to obtain three types of energy level differences. First, heterointerface calculations were carried out at the GGA level to obtain the difference between electrostatic potentials near the centre of slabs of the two phases. Second, bulk calculations provide the difference from the electrostatic potential to the valence band maximum for each phase. It should be noted that in the first calculation, we incorporated the effect of the strain due the lattice-mismatch heterojunction, however, this $\mathrm{CuGaS}_{2}: \mathrm{Cr}$ mismatch and the different semiconductors are reduced respect to the lattice-mismatch without chromium, for the $\mathrm{CuGaS}_{2}: \mathrm{Cr} / \mathrm{CuAlSe}_{2}$ the lattice mismatch is $4.72 \%$ and for the $\mathrm{CuGaS}_{2}: \mathrm{Cr} / \mathrm{ZnSe}$ is $5.9 \%$, meanwhile in the $\mathrm{CuGaS}_{2} / \mathrm{CuAlSe}_{2}$ interface, the lattice mismatch is $5.96 \%$ and for the $\mathrm{CuGaS}_{2} / \mathrm{ZnSe}$ interface is $5.68 \%$. This is due for the presence of three cationic species. For that reason we took the lattice constant and atomic position without distortion. Finally, for these undistorted cells, we have used the many body GW approximation to carried out quasiparticle energy calculations. Using this method we have predicted the electrostatic potential and the band structures more accurately.

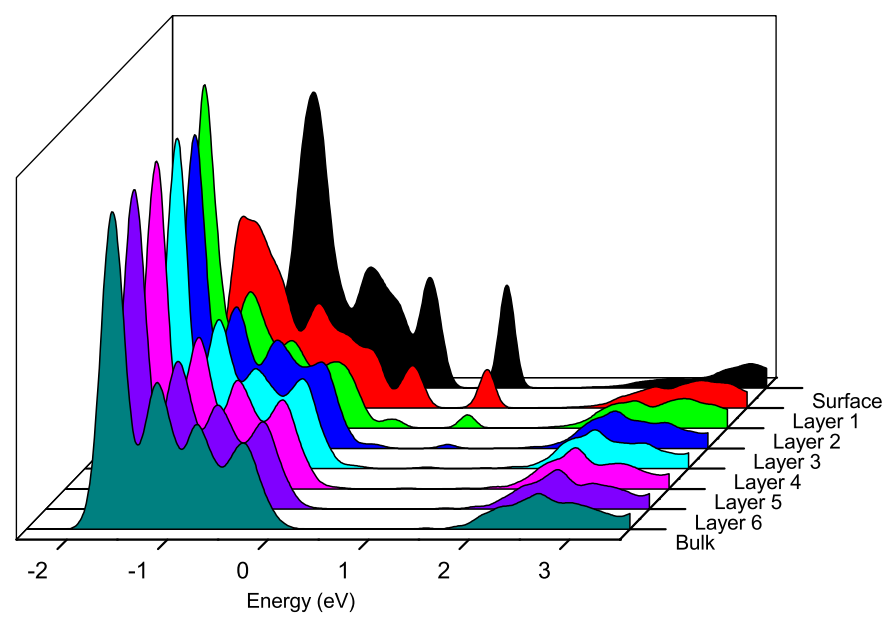

Fig. 1. Densities of states in the first seven top-layer $\mathrm{CuGaS}_{2}$ (110), compared to the bulk density of states. The zero of energy is at the Fermi level.

\section{Results}

In a previous study, we found that non-polar surfaces of $\mathrm{CuGaS}_{2}$ are technologically very important because in thin film solar cell structures are the most suitable to form cation-anion bonds across the interface, minimizing any charge accumulation. We consider for the interface the (110) plane, which is formed by an atomic layer having the stoichiometric $\mathrm{Cu}-\mathrm{S}-\mathrm{Ga}-\mathrm{S}$ composition. In this case we use slabs which stack eight such monolayers.

Fig. 1 shows the projected density of states (PDOS) for the unrelaxed surface and the first six sub-surfaces layers for the $\mathrm{CuGaS}_{2}$ (110), compared with the bulk-DOS. According to the well known DFT problem, the predicted band-gaps are underestimated and the trends in the predicted gap should be meaningful. Is important to note that the purpose of this part of the work is to determine the depth of the surface states in the chalcopyrite, which allows us to determine an integer number of $\mathrm{CuGaS}_{2}$ formula units to ensure that the effect of the interface on the level positions at the slab centre is negligible.

We see that the PDOS of the topmost atomic layer reflects the density of surface states whereas the PDOS of deeper layers become identical to the density of bulk states. Significant perturbations arising from the surface do not penetrate deeper than three layers into the bulk, which justifies using slabs containing eight atomic planes each. For the surface layer, two surface states $\left(E_{1}\right.$ and $\left.E_{2}\right)$ appear at $0.3 \mathrm{eV}$ and $1.2 \mathrm{eV}$ from the valence band. These levels correspond to $3 d$ of $\mathrm{Cu}$ and $4 p$ of $\mathrm{Ga}$, respectively. A more extensive analysis of the LDOS shows that this is a consequence of two covalent $\mathrm{Cu}-\mathrm{S}$ and $\mathrm{Ga}-\mathrm{S}$ bonds breaking in the $z$ direction of a primitive translation vector of the surface unit cell. Particularly, $\mathrm{E}_{1}$ are mostly of $d_{z^{2}}$ symmetry (where the normal to the surface is taken as $z$ direction), meanwhile the state $\mathrm{E}_{2}$ is primarily of $p_{z}$ symmetry.

From this, the created supercell contains sixteen atomic layers for both the $\mathrm{CuGaS}_{2}: \mathrm{Cr} / \mathrm{CuAlSe}_{2}$ and $\mathrm{CuGaS}_{2}: \mathrm{Cr} / \mathrm{ZnSe}$ interfaces, as it is shown in Fig. $2 \mathrm{a}$ for the interface to $\mathrm{CuAlSe}_{2}$ case. The total numbers of layers of the slab are checked to guarantee that the effects of the interfaces do not affect the electronic properties and the electrostatic potential for the slab model. Fig. 2b shows the PDOS (from the GGA calculation) for the interface and the first three subinterface layers (one of them containing the transition metal). We observe that the electronic properties of each material are maintained in the heterostructure. Surface states such as those in Fig. 1 do not appear because broken bonds are absent. In the interface layers, the effect of the lattice mismatch is observed in the edge of the valence and conduction band, where the band-gap is almost 


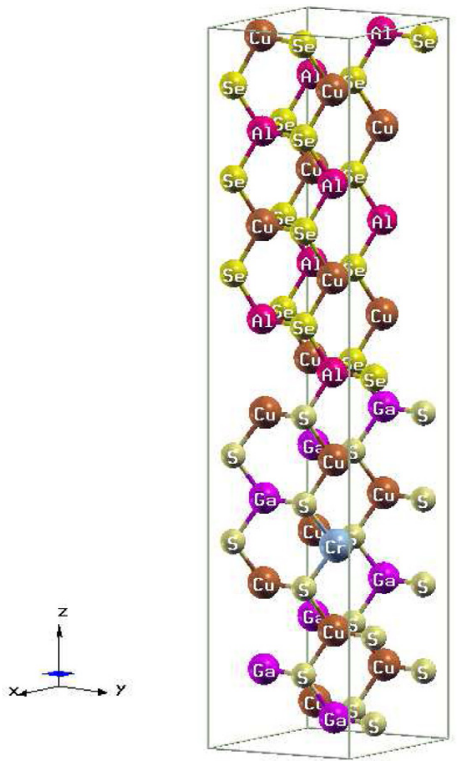

(a)

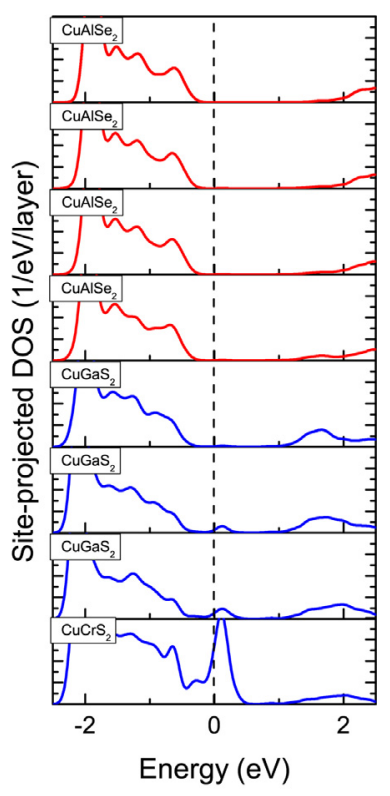

(b)

Fig. 2. (a) Structure of the supercell and (b) projected density of states as a function of the distance for the $\mathrm{CuGaS}_{2}: \mathrm{Cr} / \mathrm{CuAlSe}_{2}$ (110) interface. For the $\mathrm{CuGaS}_{2}: \mathrm{Cr} / \mathrm{ZnSe}$ interface, the supercell is constructed similarly.

equal and the bulk features converges very quickly as one moves away from the interface. For the $\mathrm{CuGaS}_{2}: \mathrm{Cr}$ interfacial layer, the hybridization from the $\mathrm{Cu} 3 d_{z^{2}}$ states with the Se $4 p_{y, z}$ orbitals of the ZnSe slab, expands the valence band and the band-gap decreases. The minimum of the conduction band in the $\mathrm{CuGaS}_{2}: \mathrm{Cr}$ interfacial layer, comes from the $4 p_{x, z}$ of the Gallium and the $4 s$ orbital of the Selenium, and pushes down into the upper part of the gap. The opposite effect is observed for the interfacial layer of CuAlSe 2 .

However, the band alignment between two materials cannot be achieved from a direct comparison of the corresponding band edges on both sides of the interfaces [23]. The reason for that is due to: (i) the lack of an common reference energy between the energy levels of the materials which comprise the heterostructure, in this case, the average electrostatic potential, and (ii) the underestimation of the band-gaps, as this affects mainly the conduction band offset.

Therefore, to calculate the band alignment between two materials, we follow the procedure introduced by Van de Walle and Martin [13], where we first compute the planar average of the potential electrostatic across the supercell, which is averaged in the region away from the interface where the electrostatic potential reaches a constant value. Second, the position of the valence band edge with respect to the average electrostatic potential is determined. To obtain the alignment, separate calculation using a highly accurate method of the electronic structure and electrostatic potential, for the corresponding bulk materials forming the interface, are required.

The valence band alignment at each interface is calculated, as explained in detail in Ref. [11], $\Delta E_{V}=\Delta E P_{\text {int }}-\Delta E P+\Delta E_{V B}$, where $\triangle E P_{\text {int }}$ is the discontinuity in this reference potential across the interfaces, $\triangle E P$ and $\Delta E_{V B}$ are the differences between the valence band edges and the electrostatic potential obtained from the two independent bulk calculations of the singles phases.

However, the conventional DFT approaches have deficiencies in describing the band-gap due to the self-interaction. Besides this approach is totally inadequate to study the electronic structure of materials where the band-gap is influenced by the hybridization of the $d$ orbitals of a transition metal with $p$ orbitals of other atom

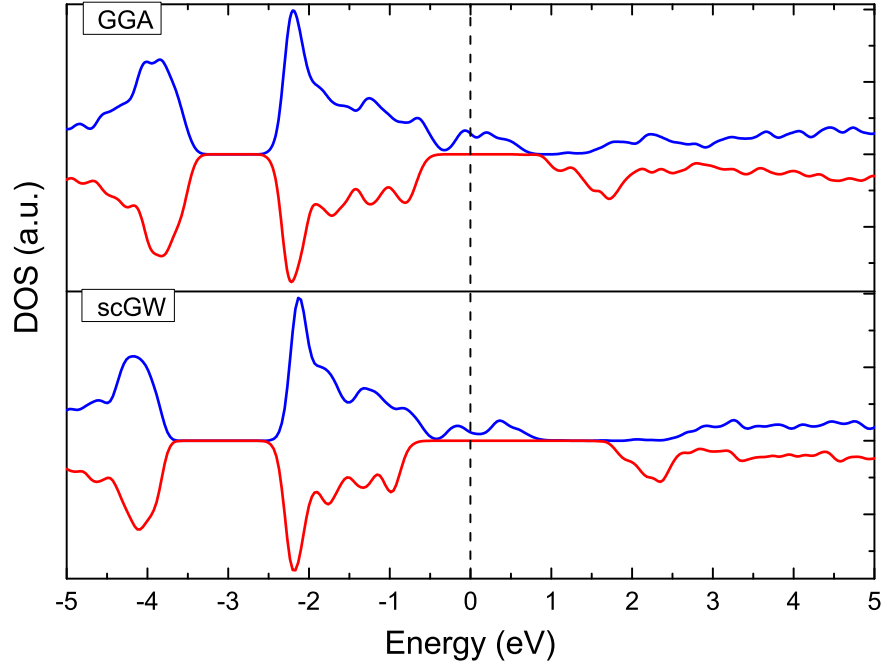

Fig. 3. Spin polarized total DOS curves obtained within GGA and scGW for the $\mathrm{CuGaS}_{2}: \mathrm{Cr}$. The dotted line indicates the Fermi level.

[24]. So we have used a self-consistent GW procedure which has been extremely successful in describing quasi-particles energies for transition metal compounds, where perturbative GW fails $[25,26]$. Another reason to go beyond DFT remains in the need to calculate excited states for application in the domain of photovoltaics, where it is necessary to evaluate quasi-particle energies, absorption spectra and exciton binding energy $[22,26]$.

Under the scheme of the scGW calculations, the band-gaps of pure $\mathrm{CuGaS}_{2}, \mathrm{CuAlSe}_{2}$ and $\mathrm{ZnSe}$ were of $2.24 \mathrm{eV}, 2.41 \mathrm{eV}$ and $2.69 \mathrm{eV}$ respectively. These results show that the standard DFT approach underestimate the band-gap $\left(0.65 \mathrm{eV}\right.$ for $\mathrm{CuGaS}_{2}, 0.87 \mathrm{eV}$ for $\mathrm{CuAlSe}_{2}$ and $1.18 \mathrm{eV}$ for $\mathrm{ZnSe}$ ), and the self-consistent quasiparticle GW calculations agrees pretty well with the experimental values $\left(2.43 \mathrm{eV}\right.$ [5], $2.49 \mathrm{eV}$ [27] and $2.82 \mathrm{eV}$ [28] for $\mathrm{CuGaS}_{2}$, $\mathrm{CuAlSe}_{2}$ and $\mathrm{ZnSe}$ respectively).

Fig. 3 shows the densities of states of $\mathrm{CuGaS}_{2}: \mathrm{Cr}$ calculated within GGA and the scGW approach. In both cases, the IB is located in the band-gap formed by spin up states from $\mathrm{Cr}$ dopant and is partially occupied with the Fermi level crossing it. At the $25 \%$ dopant concentration, the IB is located at the energy region from $-0.35 \mathrm{eV}$ to $0.73 \mathrm{eV}$, which is very wide, generating a slight overlap with the valence band, because of the weak hybridization of the $t_{2}$ states of the $\mathrm{Cr}$ with the $3 \mathrm{p}$ states of the neighbouring $\mathrm{S}$ atoms. The IB is composed of three states and according to the tetrahedral crystal field, is mainly contributed from the $t_{2}$ states, since the $\mathrm{Cr} 3 d$ states are split into the lower energy $e\left(d_{z^{2}}, d_{x^{2}-y^{2}}\right)$ states, which will contain two of the three electrons in the $3 d$ band of the (formally) $\mathrm{Cr}\left(3^{+}\right)$ion, and higher energy $t_{2}$ states $\left(d_{x y}, d_{y z}, d_{z x}\right)$ which will contain the third electron. This splitting shows how in the $\mathrm{CuGaS}_{2}: \mathrm{Cr}$ material the main band-gap is divided into two sub-bands, which is an important feature to consider when carrying out the band alignment with $\mathrm{CuAlSe}_{2}$ and $\mathrm{ZnSe}$.

For the spin down states, it can be seen that chromium does not changes the host electronic structure significantly, however, under the scGW scheme, the $e$ and $t_{2}$ states break the hybridization with the conduction band and are localized at lower energies.

In order to have a better understanding of the position of the IB, we study at the GGA level the effect of the dopant concentration at $25 \%, 12.5 \%, 6.25 \%, 4.16 \%$ and $3.125 \%$ as we see in Fig. 4 . The use of large supercells shows only a slightly change in the position of the IB. Besides, as the size of the supercell is increased, the width of the intermediate band decreases as expected.

In Table 1, we present the energetic difference between the Fermi level and the valence band maximum at different 


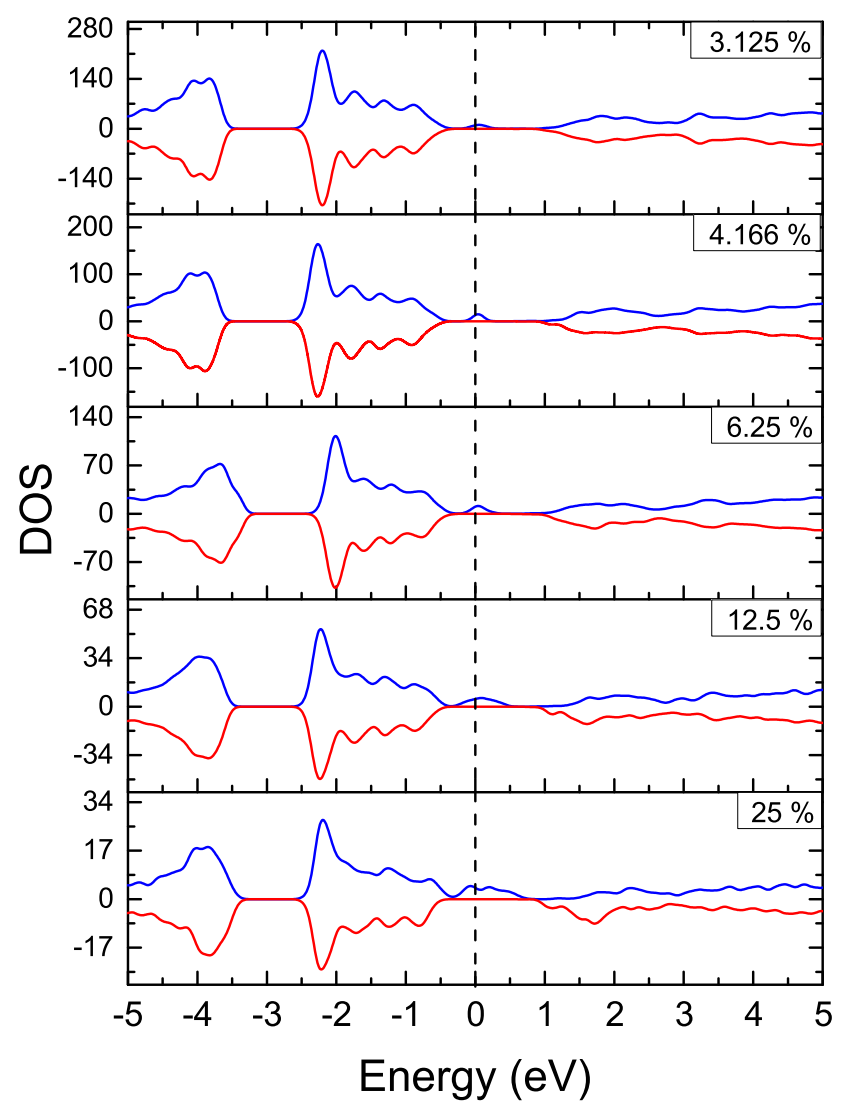

Fig. 4. DOS obtained at the GGA level for different $\mathrm{Cr}$ dopings in $\mathrm{CuGaS}_{2}$. The Fermi level is set to $0 \mathrm{eV}$.

concentrations. It is shown that this difference is similar for different concentrations. Therefore, the use of a 16 atom supercell is reasonable, since scGW is a expensive technique and scales as $n^{3}$ with the number of atoms in the unit cell, so up to date is unpractical for the study of large systems. These results suggest that the use of large supercells (less dopant concentration) isolates the IB from the valence band and the conduction band and will allow us the absorption of two photons $\left(E_{I B}-E_{V B}\right.$ and $\left.E_{C B}-E_{I B}\right)$ in addition to the photon absorbed in the host semiconductor band-gap $\left(E_{C B}-E_{V B}\right)$ without facilitating transfer of photogenerated carriers between the IB and the $\mathrm{VB}$ or $\mathrm{CB}$ via thermalization.

Therefore, for the $\mathrm{CuGaS}_{2}: \mathrm{Cr} / \mathrm{CuAlSe}_{2}$ supercell, $\Delta E P_{\text {int }}=$ $0.503 \mathrm{eV}$, and the $\Delta E P$ and $\Delta E_{V B}$ are $0.786 \mathrm{eV}$ and $-0.0069 \mathrm{eV}$ respectively. From these values the $\Delta E_{V}$ for the $\mathrm{CuGaS}_{2}: \mathrm{Cr} / \mathrm{CuAlSe}$ interface has a value of $-0.29 \mathrm{eV}$, where the valence band of the $\mathrm{CuGaS}_{2}$ : $\mathrm{Cr}$ lying in energy below the corresponding one of the CuAlSe $e_{2}$. Results for the $\mathrm{CuGaS}_{2}: \mathrm{Cr} / \mathrm{ZnSe}$ interface were also obtained in a similar way, nevertheless, the valence band offset gives $\Delta E_{V}$ equal to $0.92 \mathrm{eV}$, with $\mathrm{CuGaS}_{2}$ : $\mathrm{Cr}$ valence band above the ZnSe valence band.

Finally, from the correct description of the band-gaps and the position of the IB in the $\mathrm{CuGaS}_{2}: \mathrm{Cr}$ in conjunction with the relative position of the valence bands for each interface, the

Table 1

$E_{F}-E_{V B}(\mathrm{eV})$ calculated at GGA level for different concentrations of $\mathrm{Cr}$.

\begin{tabular}{ll}
\hline Concentration & $E_{F}-E_{V B}$ \\
\hline $25 \%$ & 0.368 \\
$12.5 \%$ & 0.383 \\
$6.25 \%$ & 0.350 \\
$4.166 \%$ & 0.377 \\
$3.125 \%$ & 0.372 \\
\hline
\end{tabular}

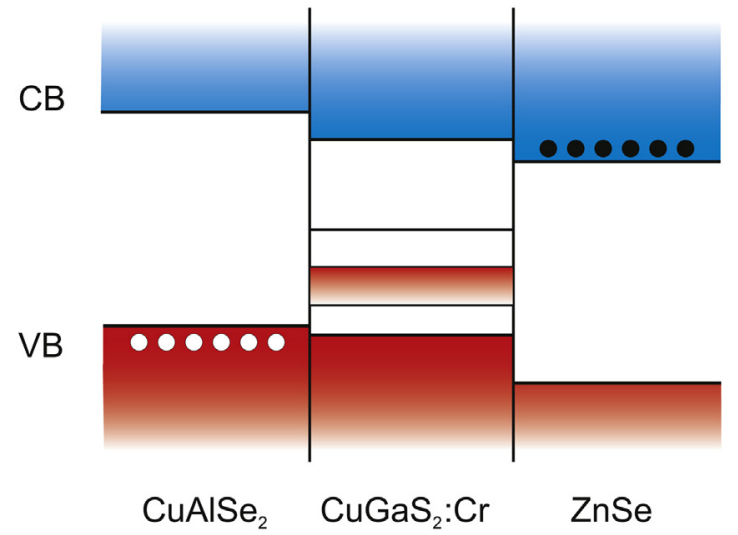

Fig. 5. Band alignment for the $\mathrm{CuGaS}_{2}: \mathrm{Cr} / \mathrm{CuAlSe}_{2}$ and $\mathrm{CuGaS}_{2}: \mathrm{Cr} / \mathrm{ZnSe}$ heterointerfaces.

resulting band alignments are shown in Fig. 5. From the figure, the $\mathrm{CuGaS}_{2}: \mathrm{Cr} / \mathrm{CuAlSe}_{2}$ interface shows a type II (staggered) alignment, with both the valence and conduction band of $\mathrm{CuGaS}_{2}: \mathrm{Cr}$ lying in energy below the corresponding ones of the CuAlSe ${ }_{2}, \Delta E_{C}=0.66 \mathrm{eV}$. This facilitates that electrons and holes to be locate on different sides of the interface, thus avoiding direct recombination. The $\mathrm{CuGaS}_{2}: \mathrm{Cr} / \mathrm{ZnSe}$ interface also exhibits a staggered band lineup, where the $\Delta E_{C}$ of $\mathrm{CuGaS}_{2}: \mathrm{Cr}$ is $0.27 \mathrm{eV}$ higher than $\mathrm{ZnSe}$.

Therefore a $\mathrm{ZnSe} / \mathrm{CuGaS}_{2}: \mathrm{Cr} / \mathrm{CuAlSe}_{2}$ setup would be ideal to efficiently separate electrons and holes generated with the help of the intermediate band. Is important to note that the position of the Fermi level in these alignments are not overlapping the valence or conduction band in the heterointerfaces.

\section{Conclusions}

In summary, we present density functional calculations for band alignment of $\mathrm{CuAlSe}_{2} / \mathrm{CuGaS}_{2}: \mathrm{Cr}$ and $\mathrm{CuGaS}_{2}: \mathrm{Cr} / \mathrm{ZnSe}$ heterointerfaces. The used scGW calculations reproduce accurately experimental band-gaps and hence correct band offsets can be obtained. The alignment, using as reference the average electrostatic potential, predicts that $\mathrm{CuGaS}_{2}: \mathrm{Cr} / \mathrm{CuAlSe} \mathrm{A}_{2}$ and $\mathrm{CuGaS}_{2}: \mathrm{Cr} / \mathrm{ZnSe}$ interfaces are from type II and possess a staggered alignment. These are the appropriate conditions to match two interfaces into a heterostructure with three semiconductors $\left(\mathrm{CuAlSe}_{2} / \mathrm{CuGaS}_{2}: \mathrm{Cr} / \mathrm{ZnSe}\right)$ so that electrons and holes photogenerated in the $\mathrm{CuGaS}_{2}: \mathrm{Cr}$ absorber layer, can be extracted selectively as desired, at both sides of the device. It is expected, that these theoretical values of $\Delta E_{V}$ and $\Delta E_{C}$ will provide further understanding of the fundamental properties of $\mathrm{CuGaS}_{2}: \mathrm{Cr} / \mathrm{CuAlSe} \mathrm{H}_{2}$ and $\mathrm{CuGaS}_{2}: \mathrm{Cr} / \mathrm{ZnSe}$ heterojunctions, which will be very useful in the design, modelling and analysis of the optoelectronic devices.

\section{Acknowledgements}

This work was partially supported by CONACyT under doctoral scholarship No. 271481. The authors thankfully acknowledge the computer resources, technical expertise and assistance provided by the Red Española de Supercomputación, the Centro de Supercomputación y Visualización de Madrid (CeSViMa) and the Laboratorio Nacional de Supercómputo del Sureste de México (LNS1).

\section{References}

[1] Solar Frontier Achieves World Record Thin-Film Solar Cell Efficiency: 22.3\%, Solar Frontier K.K. Press Release, December 8, 2015.

[2] W. Shockley, H.J. Queisser, Detailed balance limit of efficiency of pn junction solar cells, J. Appl. Phys. 32 (1961) 510-519. 
[3] A. Luque, A. Martí, Increasing the efficiency of ideal solar cells by photon induced transitions at intermediate levels, Phys. Rev. Lett. 78 (1997) 5014.

[4] A. Martí, M.D. Fuertes, A. Luque, Evaluation of the efficiency potential of intermediate band solar cells based on thin-film chalcopyrite materials, J. Appl. Phys. 103 (2008) 073706.

[5] B. Tell, J.L. Shay, H.M. Kasper, Electrical properties, optical properties, and band structure of CuGaS 2 and CuInS 2 , Phys. Rev. B 4 (1971) 2463-2471.

[6] P. Palacios, K. Snchez, J.C. Conesa, P. Wahnn, First principles calculation of isolated intermediate bands formation in a transition metal-doped chalcopyrite-type semiconductor, Phys. Status Solidi A 203 (2006) 1395.

[7] P. Palacios, K. Sánchez, J.C. Conesa, J.J. Fernández, P. Wahnón, Theoretical modelling of intermediate band solar cell materials based on metal-doped chalcopyrite compounds, Thin Solid Films 515 (2007) 6280-6284.

[8] P. Chen, M. Qin, H. Chen, Ch. Yang, Y. Wang, F. Huang, Cr incorporation in $\mathrm{CuGaS}_{2}$ chalcopyrite: a new intermediate-band photovoltaic material with wide-spectrum solar absorption, Phys. Status Solidi A 210 (2013) 1098-1102.

[9] I. Aguilera, J. Vidal, P. Wahnón, L. Reining, S. Botti, First-principles study of the band structure and optical absorption of $\mathrm{CuGaS}_{2}$, Phys. Rev. B 84 (2011) 085145.

[10] I. Aguilera, P. Palacios, P. Wahnón, Enhancement of optical absorption in Ga-chalcopyrite-based intermediate-band materials for high efficiency solar cells, Sol. Energy Sol. Cells 94 (2010) 1903-1906.

[11] J.E. Castellanos, P. Palacios, J.C. Conesa, J.J. Arriaga, P. Wahnón, Electronic band alignment at $\mathrm{CuGaS}_{2}$ chalcopyrite interfaces, Comput. Mater. Sci. 121 (2016) 79-85.

[12] F.T. Vasko, A.V. Kuznetsov, Electronic States and Optical Transitions in Semiconductor Heterostructures, Springer, New York, 1999, pp. 5-33.

[13] C.G. Van de Walle, R.M. Martin, Theoretical study of band offsets at semiconductor interfaces, Phys. Rev. B 35 (1987) 8154-8165

[14] J.P. Perdew, K. Burke, M. Ernzerhof, Generalized gradient approximation made simple, Phys. Rev. Lett. 77 (1986) 3865-3868.
[15] M. Shishkin, G. Kresse, Self-consistent GW calculations for semiconductors and insulators, Phys. Rev. B 75 (2007) 235102.

[16] G. Kresse, J. Hafner, Accurate quasiparticle spectra from self-consistent GW with vertex corrections, Phys. Rev. Lett. 99 (2007) 246403

[17] M. Shishkin, M. Marsman, G. Kresse, Ab initio molecular dynamics for liquid metals, Phys. Rev. B 47 (1993) 558-561.

[18] P.E. Blöchl, Projector augmented-wave method, Phys. Rev. B 50 (1994) 17953-17979.

[19] G. Kresse, D. Joubert, From ultrasoft pseudopotentials to the projector augmented-wave method, Phys. Rev. B 59 (1999) 1758-1775.

[20] J.E. Coulter, E. Manousakis, A. Gali, Limitations of the hybrid functional approach to electronic structure of transition metal oxides, Phys. Rev. B 88 (2013) 041107.

[21] H.J. Monkhorst, J.D. Pack, Special points for Brillouin-zone integrations, Phys. Rev. B 13 (1976) 5188-5192.

[22] I. Aguilera, J. Vidal, P. Wahnn, L. Reining, S. Botti, First-principles study of the band structure and optical absorption of $\mathrm{CuGaS}_{2}$, Phys. Rev. B 84 (2011) 085145.

[23] J. Junquera, M. Zimmer, P. Ordejón, P. Ghosez, First-principles calculation of the band offset at $\mathrm{BaO} / \mathrm{BaTiO}_{3}$ and $\mathrm{SrO} / \mathrm{SrTiO}_{3}$ interfaces, Phys. Rev. B 67 (2003) 155327

[24] F. Bruneval, N. Vast, L. Reining, Effect of self-consistency on quasiparticles in solids, Phys. Rev. B 74 (4) (2006) 045102.

[25] S.V. Faleev, M. van Schilfgaarde, T. Kotani, All-electron self-consistent GW approximation: application to $\mathrm{Si}, \mathrm{MnO}$, and NiO, Phys. Rev. Lett. 93 (12) (2004) 126406

[26] M. van Schilfgaarde, T. Kotani, S.V. Faleev, Quasiparticle self-consistent GW theory, Phys. Rev. Lett. 96 (22) (2006) 226402.

[27] Y. Shim, K. Hasegawa, K. Wakita, N. Mamedov, $\mathrm{CuAl}_{1-x} \mathrm{In}_{x} \mathrm{Se}_{2}$ solid solutions: dielectric function and inter-band optical transitions, Thin Solid Films 517 (4) (2008) 1442-1444.

[28] K.K. Ng, S.M. Sze, Physics of Semiconductors Devices, Wiley-VCH Verlag GmbH, Berlin, 2007. 